\title{
Manejo da cárie relacionada à radiação em pacientes oncológicos de cabeça e pescoço: Evidência científica
}

\author{
Management of radiation-related caries in head and neck cancer patients: Scientific evidence \\ Manejo de la caries relacionada con la radiación en pacientes con cancer de cabeza y cuello: \\ Evidencia científica
}

Recebido: 02/06/2021 | Revisado: 13/06/2021 | Aceito: 14/06/2021 | Publicado: 30/06/2021

Renata Borges Rodrigues ORCID: https://orcid.org/0000-0003-2846-4934 Universidade Federal de Uberlândia, Brasil E-mail: renataborges4@hotmail.com

Andressa Cardoso Amorim Souza ORCID: https://orcid.org/0000-0001-6885-3534 Universidade Federal de Uberlândia, Brasil E-mail: andress.amorim@ hotmail.com

Allyne Jorcelino Daloia de Carvalho ORCID: https://orcid.org/0000-0002-9231-0895 Universidade Federal de Uberlândia, Brasil E-mail: allyne.carvalho@hotmail.com

Camila de Carvalho Almança Lopes ORCID: https://orcid.org/0000-0001-9313-9818 Universidade Federal de Uberlândia, Brasil

E-mail: camila.calopes@gmail.com

Rafael Resende de Miranda

ORCID: https://orcid.org/0000-0002-5510-2747 Universidade Federal de Uberlândia, Brasil

E-mail: rafaelresendemiranda@gmail.com

Dhiancarlo Rocha Macedo

ORCID: https://orcid.org/0000-0002-9241-5187 Universidade Federal de Uberlândia, Brasil E-mail: dhiancarlo.macedo@ufu.br

Luiz Fernando Barbosa de Paulo ORCID: https://orcid.org/0000-0003-1146-0777 Universidade Federal de Uberlândia, Brasil E-mail: luizfbpaulo@gmail.com

Cizelene do Carmo Faleiros Veloso Guedes ORCID: https://orcid.org/0000-0001-7128-7537

Universidade Federal de Uberlândia, Brasil E-mail: cizelene@ hotmail.com

Veridiana Resende Novais

ORCID: https://orcid.org/0000-0001-9017-2946

Universidade Federal de Uberlândia, Brasil E-mail: veridianaresende@ @otmail.com

\begin{abstract}
Resumo
Pacientes diagnosticados com neoplasias malignas na região de cabeça e pescoço frequentemente são tratados por meio de radioterapia. Apesar da eficácia em erradicar células cancerígenas, a radiação ionizante pode atingir tecidos sadios próximos ao tumor acarretando efeitos indesejáveis ao paciente como hipossalivação, xerostomia, mucosites, necrose de tecidos moles e duros e cárie relacionada à radiação (CRR). A CRR é uma doença multifatorial e altamente destrutiva, que compromete a função oral do paciente. O manejo de pacientes com CRR está se tornando cada vez mais comum na prática odontológica, visto que os índices de cura de neoplasias malignas têm sido maiores com os avanços da medicina. Nesse contexto, o presente trabalho objetivou discutir os principais fatores relacionados ao desenvolvimento da CRR, assim como as dificuldades e limitações encontradas no seu tratamento e controle. Foi realizada uma revisão de literatura de caráter narrativo e qualitativo, a partir do levantamento de artigos científicos encontrados nas bases de dados Google Scholar, Pubmed e SciELO, sem restrição de ano de publicação e idioma. Para isto, foram utilizadas as palavras-chave: Cárie Dentária; Dentina; Esmalte Dentário; Neoplasias Bucais; Radioterapia. Conclui-se que não há um protocolo bem definido de tratamento da CRR na literatura, sendo o processo restaurador bastante desafiador ao cirurgião-dentista. Enfatiza-se a necessidade de melhor detalhamento metodológico
\end{abstract}


dos trabalhos avaliados e mais estudos sobre o tema no intuito de estabelecer um protocolo clínico eficaz, buscando, sobretudo, restabelecer saúde e melhorar qualidade de vida.

Palavras-chave: Cárie dentária; Dentina; Esmalte dentário; Neoplasias bucais; Radioterapia.

\begin{abstract}
Patients diagnosed with malignant neoplasms in the head and neck region are often treated by radiotherapy. Despite the efficiency in eradicating cancer cells, ionizing radiation can reach healthy tissues close to the tumor, causing undesirable effects to the patient, such as hyposalivation, dry mouth, mucositis, soft and hard tissue necrosis and radiation-related caries (RRC). RRC is a multifactorial and highly destructive disease, which compromises the patient's oral function. The management of patients with RRC is becoming increasingly common in dental practice, since the cure rates for malignant neoplasms have been higher with the advances in current medicine. In this context, the present study aims to discuss the main factors related to the development of RRC, as well as the difficulties and limitations found in its treatment and control. A narrative and qualitative literature review was carried out, based on a survey of scientific articles found in the Google Scholar, Pubmed and SciELO databases, with no restriction on the year of publication and language. For this, the following keywords were used: Dental Caries; Dentin; Dental Enamel; Mouth Neoplasms; Radiotherapy. It is concluded that there is no well-defined protocol for the treatment of RRC in the literature, and the restorative process is quite challenging for dentists. It emphasizes the need for better methodology details of the study carried out and more studies on the subject in order to establish an effective clinical protocol for these patients, seeking, above all, to restore health and improve quality of life.
\end{abstract}

Keywords: Dental caries; Dentin; Dental enamel; Mouth neoplasms; Radiotherapy.

\title{
Resumen
}

Apesar de la eficacia em la erradicatión de células cancerosas, la radioterapia de cabeza y cuello puede llegar a los tejidos sanos cercanos al tumor, provocando efectos indeseables en el paciente, como hiposalivación, sequedad de boca, mucositis, necrosis de tejidos y caries relacionadas con la radiación (CRR). La CRR es una enfermedad multifactorial y altamente destructiva, que compromete la función oral del paciente. El manejo de pacientes con CRR es cada vez más común en la práctica odontológica, ya que las tasas de curación de neoplasias malignas han sido mayores con los avances de la medicina. En este contexto, el presente estudio tiene como objetivo discutir los principales factores relacionados con el desarrollo de la CRR, así como las dificultades y limitaciones encontradas en su tratamiento y control. Se realizó una revisón de literatura narrativa y cualitativa, a partir de una encuesta de artículos científicos encontrados en la bases de datos Google Scholar, Pubmed y SciELO, sin restricción de año de publicación e idioma. Para ello, se utilizaron las siguientes palabras clave: Caries Dental; Dentina; Esmalte Dental; Neoplasias de la Boca; Radioterapia. Se concluye que no existe un protocolo bien definido para el tratamiento de la CRR en la literatura, y el proceso de restauración es bastante desafiante para los dentistas. Destaca la necesidad de um mejor detalle metodológico del trabajo realizado y más estudios sobre el tema con el fin de establecer un protocolo clínico eficaz, buscando, sobre todo, restaurar la salud y mejorar la calidad de vida.

Palabras clave: Caries dental; Dentina; Esmalte dental; Neoplasias de la boca; Radioterapia.

\section{Introdução}

Câncer de cabeça e pescoço é o nome dado a um grupo heterogêneo de neoplasias malignas que acometem vários locais, incluindo nasofaringe, seios paranasais, cavidade oral, orofaringe e laringe (De Felice et al., 2018). Classifica-se como câncer de boca aqueles que tenham como localização primária os lábios, a cavidade oral, as glândulas salivares e a orofaringe (C00-C10). No Brasil, estimam-se 15.190 novos casos de câncer na cavidade oral para cada ano do triênio 2020-2022, sendo, portanto, uma preocupação para a saúde pública (INCA, 2020).

A radioterapia é uma modalidade terapêutica baseada no uso da radiação ionizante para tratamento de neoplasias malignas de forma exclusiva ou associada à cirurgia e/ou quimioterapia (Buglione et al., 2016; Moore et al., 2020). É um tratamento altamente eficaz para o controle de tumores, entretanto também atinge tecidos sadios da cavidade oral, como glândulas salivares, ossos, mucosa oral, dentes e músculos, o que resulta em reações adversas que afetam significativamente a qualidade de vida dos pacientes (Jawad et al., 2015; Kirka et al., 2017; Bhandari et al., 2020; Moore et al., 2020; Cartaxo et al., 2021). Na cavidade oral, essas reações podem resultar em mucosites, diminuição da salivação, xerostomia, disfagia, disgeusia, trismo, perda progressiva de inserção periodontal, necrose de tecido mole, osteorradionecrose e cárie dentária (Jawad et al., 2015; Buglione et al., 2016; Moore et al., 2020; Cartaxo et al., 2021). 
A cárie relacionada à radiação (CRR) é uma lesão que acomete os pacientes submetidos à radioterapia na região de cabeça e pescoço, quando o campo de radiação do tumor envolve a cavidade oral (Jawad et al., 2015; Moore et al., 2020). Assim como a cárie convencional, a CRR possui etiologia multifatorial. A hipossalivação, causada por danos da radiação às glândulas salivares, faz com que o ambiente oral se torne mais ácido (Noone et al., 2017). Como consequência da alteração na quantidade e na qualidade da saliva, os pacientes pós-radioterapia podem apresentar desconforto oral e, com isso, optarem por uma dieta baseada em alimentos líquidos e pastosos. O meio oral se torna altamente cariogênico: pouca saliva, ambiente ácido e alimentação rica em carboidratos (Jawad et al., 2015). Além disso, as propriedades químicas e mecânicas dos dentes atingidos pela radiação ionizante são evidentemente alteradas, resultando em enfraquecimento e destruição dos mesmos (Soares et al., 2010; Gonçalves et al., 2014; Reed et al., 2015; Novais et al., 2016; Qing et al., 2016; Rodrigues et al., 2018; Lopes et al., 2018; Miranda et al., 2019, Velo et., 2018; Lu et al., 2019; Miranda et al., 2021). Dessa forma, a cárie desenvolvida nesses pacientes evolui mais rapidamente, podendo resultar na perda do elemento dentário em um curto intervalo de tempo.

Ainda existem muitas limitações quanto ao diagnóstico, prevenção e tratamento da CRR. Não há um protocolo preventivo e restaurador bem definido na literatura, sobre a conduta terapêtica mais adequada, o que torna o manejo e o tratamento do paciente após a radioterapia extremamente desafiadores para os cirurgiões-dentistas. Nesse contexto, o objetivo deste trabalho é discutir os principais fatores relacionados ao desenvolvimento da CRR, assim como as dificuldades encontradas no seu controle e tratamento.

\section{Metodologia}

Trata-se de uma pesquisa de revisão de literatura narrativa. A busca da literatura ocorreu de novembro de 2020 a março de 2021, utilizando as seguintes palavras-chave: "dental caries"; "enamel”; "dentin"; head and neck neoplasms; "radiotherapy" e os operadores booleanos "AND" e "OR" para a associação entre estes. O levantamento dos artigos científicos foi realizado nas bases de dados Google Scholar, Pubmed e SciELO. Para a seleção dos estudos, utilizou-se os seguintes critérios de inclusão: artigos originais, sem restrição de idioma e sem delimitação de período de publicação, com base na relevância e significância clínica. Os critérios de exclusão foram: artigos indisponíveis na íntegra e que não abordassem a temática. Assim, ao final, foram selecionados 61 artigos para análise e discussão.

\section{Resultados e Discussão}

\section{Fatores predisponentes da CRR}

Amplamente aceita como uma doença complexa e multifatorial, a CRR é predominantemente descrita como consequência do efeito direto e indireto da radiação. A hipossalivação induzida pela radiação, as alterações nas propriedades do esmalte e da dentina, a alteração da microflora oral, e a composição alterada da saliva associadas a fatores indiretos como mucosites, mudanças da dieta, disfagia e trismo, com ênfase reduzida nas práticas de higiene bucal, em sinergia, são considerados como parte da patogênese da CRR. (Vissink et al., 2003; Silva et al.,2009; Gupta et al., 2015, Jawad et al., 2015; Alves et al., 2020; Moore et al., 2020). A seguir são apresentados como cada fator contribui para o desenvolvimento e progressão da CRR.

\section{Alterações salivares}

O protocolo radioterápico normalmente preconizado para pacientes oncológicos de cabeça e pescoço baseiam-se numa dose total de radiação de 50 a 70 Gy, fracionadas em 1,8 a 2 Gy diários, num período de 5 a 7 semanas (Qing et al., 2016; Bhandari et al., 2020). Caso as glândulas salivares se encontrem localizadas no campo de radiação, elas poderão sofrer 
danos diretos nas suas células glandulares, resultando na redução do fluxo salivar, evidente após aproximadamente 20 Gy (Noone et al., 2017; Muller et al., 2019). A diminuição efetiva da quantidade do fluxo salivar é denominada hipossalivação, enquanto a xerostomia é compreendida como a sensação subjetiva de boca seca, consequente ou não da alteração da função das glândulas salivares. A diminuição do fluxo salivar ocorre, em maior parte, devido ao dano nas glândulas parótidas e submandibulares, responsáveis pela produção de aproximadamente $80 \%$ da saliva (Wu \& Leung, 2019). Esta condição se desenvolve logo após o início da radioterapia, progredindo durante todo o tratamento e até 1 ano após seu término, podendo regredir posteriormente (Muller et al., 2019; Alves et al., 2020; Kawashita et al., 2020). No entanto, doses cumulativas de radiação acima de 30 Gy são capazes de causar xerostomia severa e permanente, tornando-se assim um problema grave para o paciente oncológico (Muller et al., 2019; Bhandari et al., 2020). Pacientes com xerostomia queixam-se de desconforto bucal, perda do paladar, dificuldades na fala, na deglutição, no sono e comprometimento da ingestão nutricional (Lou et al., 2018; Muller et al., 2019; Alves et al., 2020; Lan et al., 2020). A ausência de saliva deixa a mucosa oral com aparência seca, atrófica, pálida ou hiperêmica. Diante dessas alterações, o paciente geralmente opta por alimentos macios e ricos em carboidratos e apresenta dificuldade em realizar uma higienização bucal eficiente (Vissink et al., 2003; Jawad et al., 2015).

Ademais, a saliva mantém a homeostase da cavidade oral através de várias funções como lubrificação, capacidade tampão e atividade antimicrobiana (Roblegg et al., 2019). Com a diminuição do fluxo, há um aumento da viscosidade, alteração dos sistemas antimicrobianos e uma redução da atividade da amilase e do bicarbonato (Noone et al., 2017; Muller et al., 2019). O pH salivar que normalmente é em torno de 7,0 cai para 5,0 em pacientes pós-radioterapia, o que contribui no processo de desmineralização das estruturas dentárias (Noone et al., 2017). Dessa forma, a redução quantitativa da saliva levará a alteração de concentrações iônicas e suas propriedades antibacterianas. Isso resultará na alteração das colônias de microorganismos com aumento das bactérias cariogênicas, como Streptococcus mutans e Lactobacillus species (Noone et al., 2017; Muller et al., 2019; Comodo et al., 2020). Portanto, a ausência de saliva contribui significativamente para o aparecimento e desenvolvimento da CRR, ficando evidente que as alterações salivares são preocupantes no manejo odontológico do paciente pós-radioterapia de cabeça e pescoço. A hipossalivação e a xerostomia fazem com que esses pacientes tenham dificuldade para falar, deglutir, sentir o sabor dos alimentos e até mesmo dormir. Tais alterações impactam diretamente na qualidade de vida desses pacientes e na sua interação social.

\section{Alterações nas propriedades do esmalte e da dentina}

A radioterapia é capaz de alterar a composição química e as propriedades mecânicas dos tecidos dentais duros esmalte e dentina (Soares et al., 2010; Gonçalves et al., 2014; Reed et al., 2015; Qing et al., 2016; Rodrigues et al., 2018; Velo et al., 2018; Miranda et al., 2019; Lu et al., 2019; Fonseca et al., 2020; Muñoz et al., 2020; Lopes et al.; 2021; Miranda et al., 2021). O esmalte consiste em, aproximadamente, $96 \%$ de matriz inorgânica e $4 \%$ de porção orgânica e água, enquanto a dentina apresenta $70 \%$ de conteúdo inorgânico, $20 \%$ orgânico e 10\% de água (Teruel et al., 2015). A radiação ionizante atua de forma direta sobre a estrutura dental, sendo vistas alterações nos prismas e na zona interprismática do esmalte, gerando acentuada desmineralização subsuperficial, somadas a desorganização da dentina peritubular e intertubular, que pode ser associada à diminuição da microdureza da dentina (Palmier et al., 2020).

O esmalte é altamente rígido e estruturalmente organizado em prismas ricos em hidroxiapatita, que representam o seu conteúdo mineral (Teruel et al., 2015). A radiação ionizante altera principalmente a região interprismática, local onde se localiza a maior parte orgânica do tecido (Santin et al., 2015). Microscopicamente, esta região apresenta um padrão de esmalte altamente poroso, evidenciando considerável alteração morfológica do tecido nesta área após a radiação (Madrid et al., 2017). Com as alterações nessa região, o esmalte torna-se menos resistente à fratura e apresenta alterações nas suas propriedades mecânicas, microdureza e módulo de elasticidade, o que torna o tecido mais friável (Santin et al., 2015). Além disso, ocorrem 
mudanças na composição química do tecido, com alterações na matriz orgânica e componentes minerais, aumentando a solubilidade do esmalte e favorecendo o processo de desmineralização (Lopes et al., 2018; Lu et al., 2019; Miranda et al., 2021).

Já a dentina é um tecido composto por 70\% de matéria inorgânica (hidroxiapatita), 18\% de matriz orgânica e 12\% de água (Teruel et al., 2015). A matriz orgânica é composta principalmente por fibrilas colágenas que sofrem alterações estruturais pela radioterapia, levando à sua fragmentação (Gonçalves et al., 2014). Essas alterações no colágeno interferem na interação da matriz orgânica com os cristais de hidroxiapatita, diminuindo, por conseguinte, a dureza e o módulo de elasticidade da dentina (Rodrigues et al., 2018). Alterações na porção inorgânica também ocorrem, por meio de troca de íons fosfato-carbonato na hidroxiapatita e diminuição da relação entre cálcio e fósforo (Velo et al., 2018), tornando o tecido mais solúvel em meio ácido, assim como acontece no esmalte (Lopes et al., 2018; Miranda et al., 2021).

Outra região que se apresenta bastante instável após a radioterapia é a junção amelodentinária (JAD), conector natural entre o esmalte e a dentina e desempenha um papel crítico na manutenção da integridade biomecânica do dente ( $\mathrm{Lu}$ et al., 2019; Fonseca et al., 2020). O colágeno é afetado pela radiação ionizante e isso implica diretamente na instabilidade da junção (McGuire et al., 2014). Essa instabilidade pode causar o descolamento do esmalte, num processo chamado delaminação, com consequente exposição da dentina subjacente (Reed et al., 2015; Lu et al., 2019; Kudkuli et al., 2020). Clinicamente, o dente apresenta-se com aspecto quebradiço, com lascas de esmalte que podem ser facilmente removidas. $\mathrm{O}$ dano estrutural pode chegar a ser tão grande que causa fraturas coronárias e até mesmo radiculares.

\section{Características clínicas da CRR}

Pode-se diferenciar CRR de lesões de cárie convencionais em relação à sua localização, aparência e progressão. Os primeiros sinais da CRR podem surgir aproximadamente 3 meses após o início da radioterapia e progredir ao longo de anos (Bhandari et al., 2020). Geralmente, a CRR apresenta alteração na cor e translucidez do esmalte, podendo ser de coloração marrom escura à preta, com progressão rápida e destrutiva. É caracterizada inicialmente por delaminação e fraturas do esmalte de forma parcial ou completa, com consequente exposição da dentina subjacente, podendo progredir até a amputação das coroas dentárias e/ou a perda total da dentição do paciente (Walker et al., 2011; Lu et al., 2019; Bhandari et al., 2020; Palmier et al., 2020). Curiosamente, essas lesões de cárie geralmente não estão associadas a dor intensa (Vissink et al., 2003; Kielbassa et al.,

2006).

Quanto à localização, enquanto a cárie convencional ocorre em regiões de fossas, fissuras e nas superfícies proximais dos dentes, que são regiões historicamente relacionadas ao acúmulo de placa bacteriana, a CRR tende a ocorrer na região cervical, próximo à junção entre coroa e raiz, em pontas de cúspides e bordas incisais de dentes anteriores (Jawad et al., 2015; Noone et al., 2017; Lu et al., 2019; Bhandari et al., 2020) (Figuras 1 e 2). Esses locais são expostos a grandes cargas oclusais e de flexão associadas e são considerados mais resistentes à deterioração dentária causada pela cárie convencional por serem locais de fácil higienização. Entretanto, no caso de dentes de pacientes submetidos à radioterapia na região de cabeça e pescoço, cujas propriedades mecânicas apresentam-se alteradas, essas regiões se tornam menos resistentes aos esforços mastigatórios. Associado a estes fatores, há também um aumento de metaloproteinases da matriz na JAD, que pode causar a degradação de proteínas, favorecendo o aparecimento de trincas e a delaminação do esmalte nesta região (Palmier et al., 2020). 
Research, Society and Development, v. 10, n. 7, e47810716733, 2021

(CC BY 4.0) | ISSN 2525-3409 | DOI: http://dx.doi.org/10.33448/rsd-v10i7.16733

Figura 1. Aspecto clínico de cáries relacionadas à radiação. Observa-se uma completa perda do esmalte cervical no dente 42 (delaminação). Nos demais dentes, observam-se mudanças de cor da dentina e aspecto geral de desmineralização.

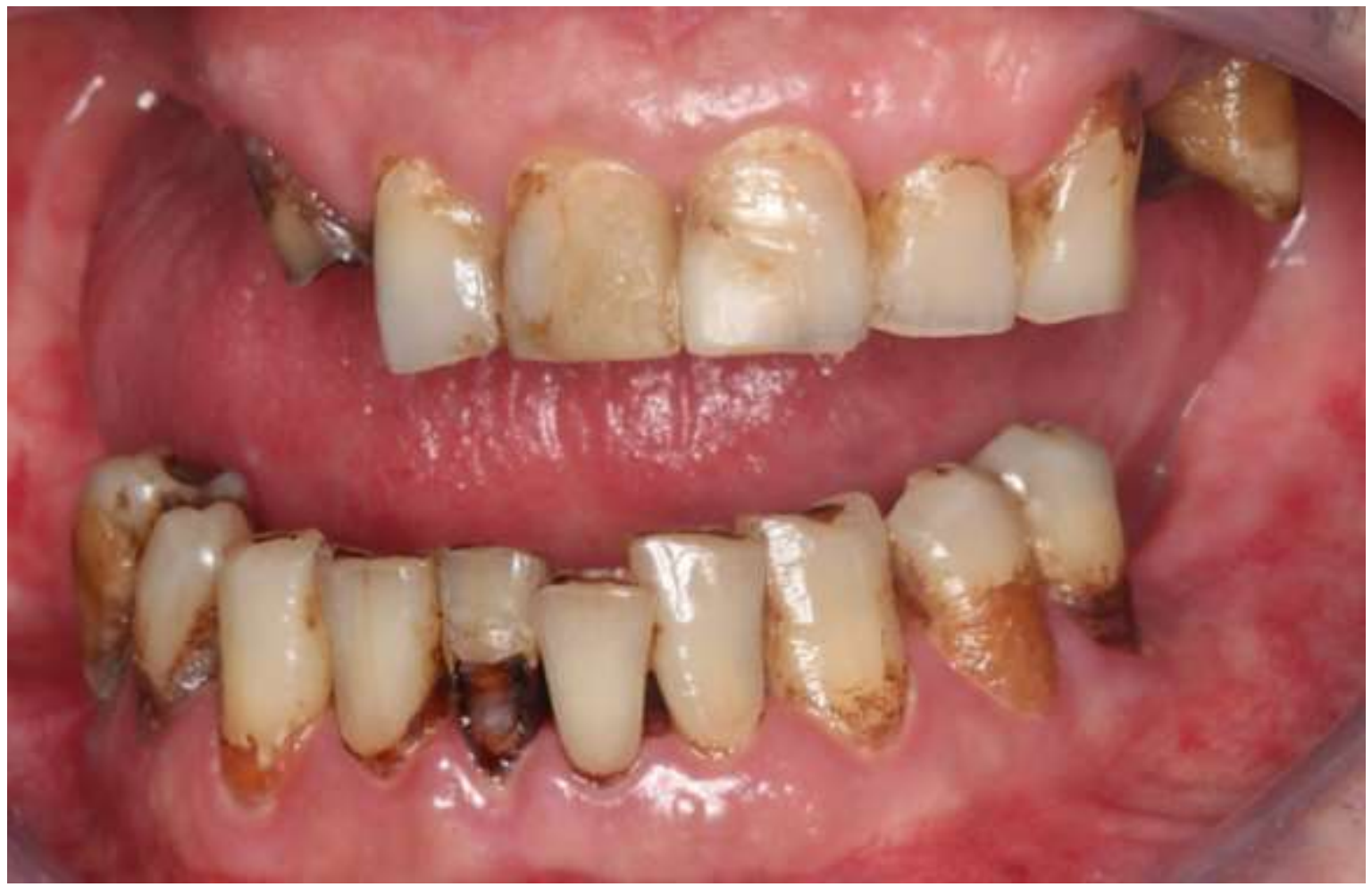

Fonte: Autores. 
Figura 2. Vista oclusal da arcada superior (A) e da arcada inferior (B). Pode-se observar os desgastes incisais e nas pontas de cúspides dos pré-molares. Na arcada inferior observa-se também o acometimento da CRR na região cervical lingual de incisivos e caninos
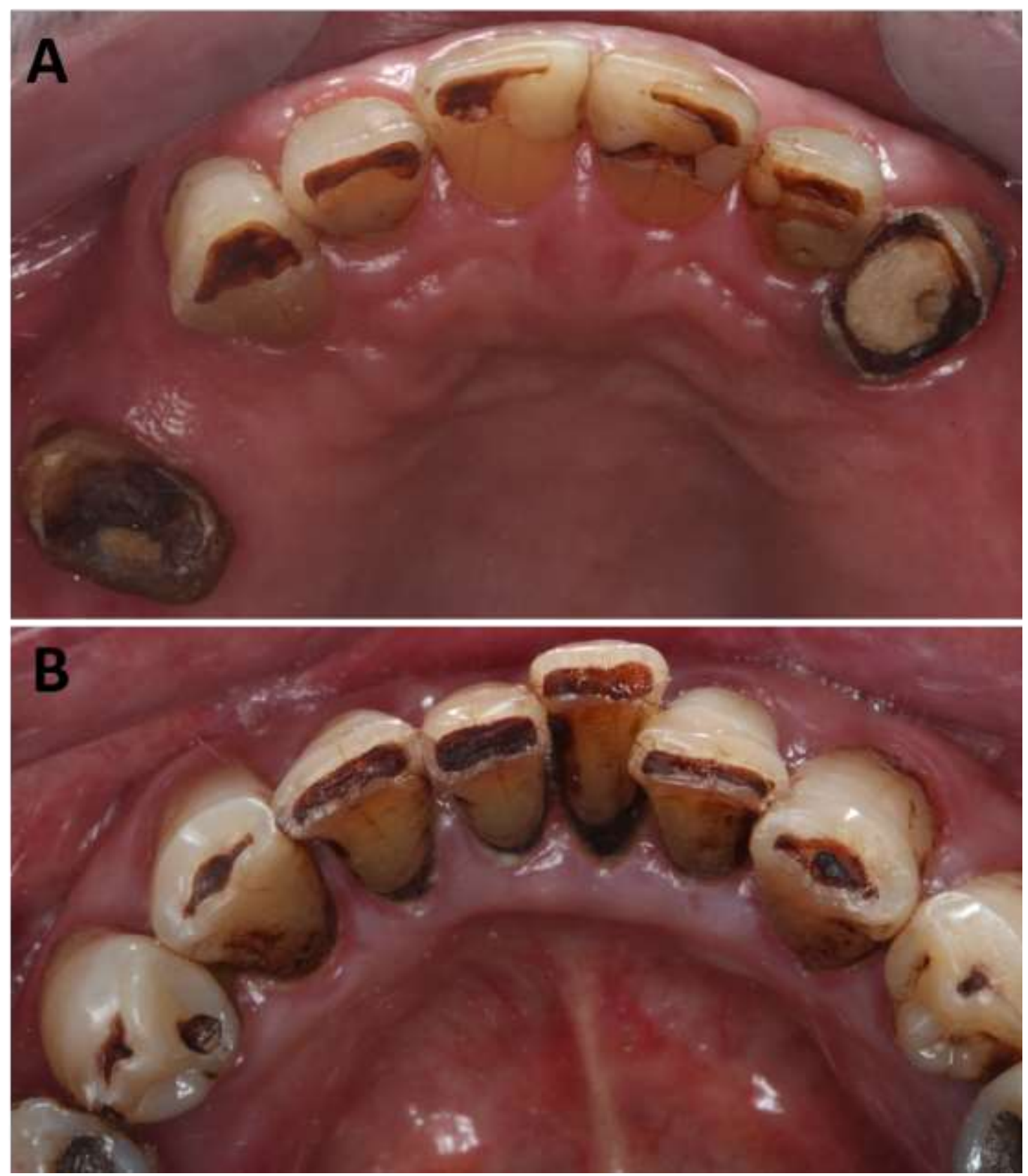

Fonte: Autores.

O local onde a CRR progride mais rapidamente é na região cervical. Além das alterações mecânicas e da dissipação das cargas oclusais nessa região, associa-se também a ausência da proteção salivar e o acúmulo de placa bacteriana. Dessa forma, pode-se afirmar que uma somatória de fatores torna essa região especialmente preocupante. A CRR neste local inicia-se nas faces vestibular e lingual dos dentes, podendo se estender por toda a região cervical (Vissink et al., 2003). Ainda na fase inicial, pode ocorrer microfissuras na região da JAD, fazendo com que haja acúmulo de bactérias neste local (Pioch et al., 1992; Walker et al., 2011). A adoção de uma dieta pastosa e cariogênica, associada à higienização deficiente, falta de saliva e alterações intrínsecas nos tecidos irradiados fazem com que a evolução da CRR seja rápida, podendo atingir regiões mais profundas causando a amputação completa da coroa dental (Vissink et al., 2003; Walker et al., 2011; Noone et al., 2017; 
Bhandari et al., 2020; Moore et al., 2020). A possível perda de um elemento dentário pode acarretar outros problemas. A exodontia nesses pacientes pós-radioterápicos é um procedimento que requer cuidados específicos, considerando as alterações do tecido ósseo irradiado e o provável risco de osteorradionecrose (Jawad et al., 2015; Kawashita et al., 2020; Moore et al., 2020).

Além disso, os sintomas causados pelos efeitos colaterais crônicos do tratamento antineoplásico de cabeça e pescoço podem contribuir indiretamente para o início da CRR e sua progressão, compondo um panorama muito mais complexo em termos de patogênese da cárie (Gouvêa et al., 2020). Dessa forma, os protocolos atuais de prevenção e tratamento da CRR devem levar em consideração esse agrupamento mais amplo de toxicidades associado à radioterapia (Gouvêa et al., 2020) (Figura 3).

Figura 3. Esquema ilustrativo relacionando efeitos diretos e indiretos no desenvolvimento da cárie relacionada à radiação.

\section{Efeitos indiretos da radioterapia}

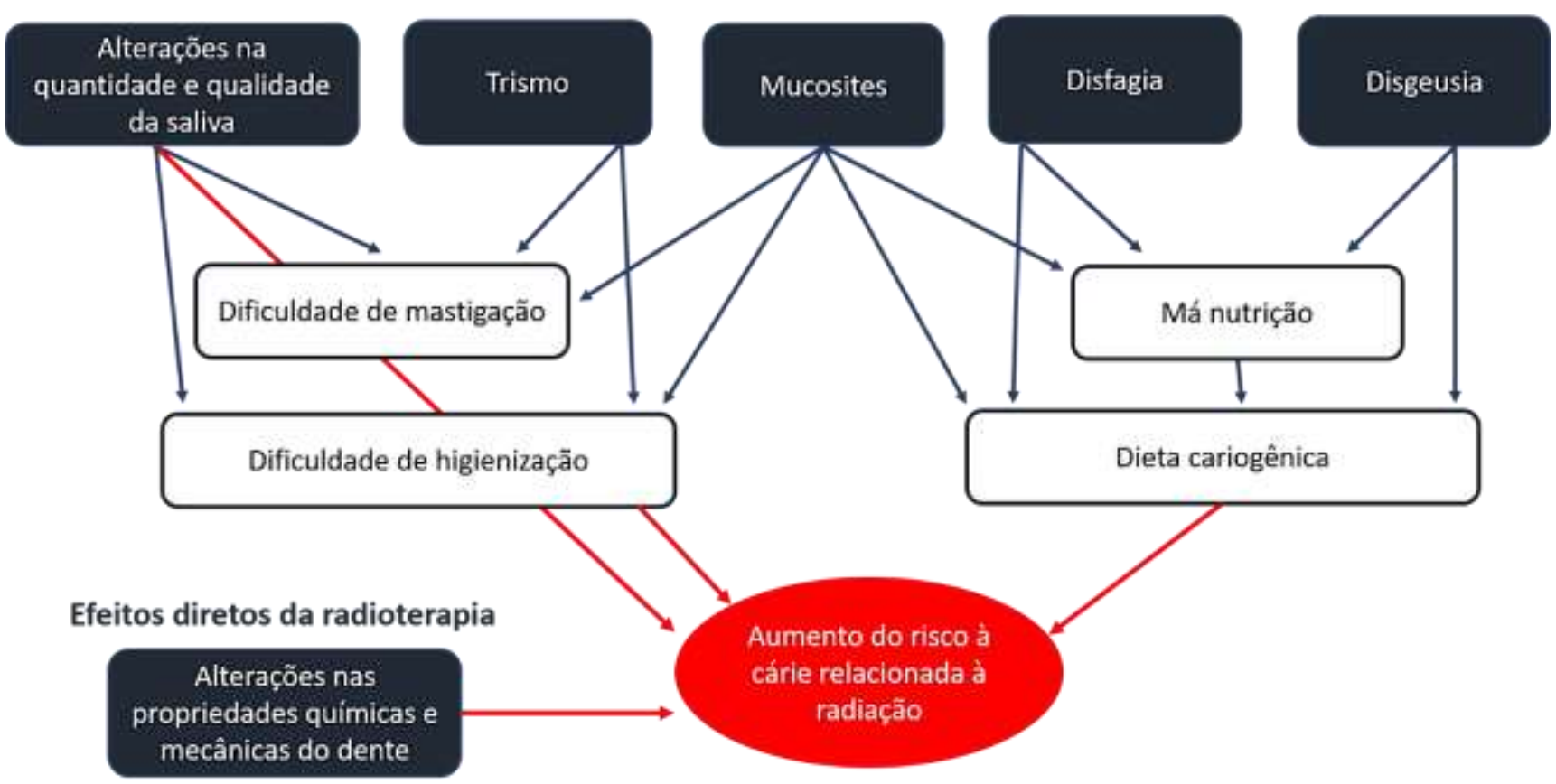

Fonte: Adaptado de Moore et al. (2020).

\section{Prevenção e tratamento da CRR}

É fundamental que o paciente oncológico passe pela avaliação de um cirurgião-dentista previamente à radioterapia. O profissional poderá orientar o paciente quanto aos cuidados de higiene oral e realizar os tratamentos odontológicos preventivos e curativos necessários. Restaurações de lesões cariosas e não-cariosas, trocas de restaurações insatisfatórias e quaisquer outros procedimentos odontológicos como tratamentos endodônticos, periodontais e cirúrgicos, idealmente devem ser realizados antes da radioterapia (Jawad et al., 2015; Palmier et al., 2020). Claro que isso dependerá de outros fatores como o estadiamento do tumor e o estado de saúde geral do paciente.

\section{Prevenção de nível individual}

Previamente à radioterapia, o paciente deverá ser instruído quanto à higienização e importância do uso constante do fluoreto assim que iniciar o tratamento oncológico radioterápico (Buglione et al., 2016; Lopes et al., 2018). Recomenda-se a escovação atraumática com uso de escova de cerdas macias e limpeza interdental com fio dental e escovas específicas (Palmier 
et al., 2020). Os dados encontrados na literatura apoiam claramente o uso de fluoreto como um dos pilares do atendimento odontológico preventivo da CRR (Epstein et al.,1996). Tanto a frequência de exposição quanto a concentração de flúor são cruciais. A escovação com pastas de 5000 ppm de flúor, duas vezes ao dia, pode auxiliar na prevenção (Noone et al., 2017). Além disso, uma quantidade do tamanho de uma ervilha, ou géis de flúor 1\%, podem ser usados em moldeiras por 30 minutos diários (Noone et al., 2017; Bhandari et al., 2020). Em casos que impossibilitem o uso das moldeiras, a prevenção com 0,05\% de fluoreto de sódio (sem álcool) na forma de enxaguante de uso diário, por um minuto, é uma alternativa (Noone et al., 2017). Além da prevenção da CRR, o uso do flúor durante o período da radioterapia auxilia na manutenção das propriedades intrínsecas do esmalte, além de fornecer benefícios ao processo de remineralização das lesões iniciais de cárie (Soares et al., 2011; Lopes et al., 2018; Bhandari et al., 2020; Kawashita et al., 2020; Palmier et al., 2020).

Infelizmente, a eficácia do fluoreto pode ser limitada nessa população devido à redução de íons de cálcio e fosfato secundária à hipossalivação. A remineralização não pode ocorrer se o grau de saturação de cálcio e fosfato na saliva relacionados com o mineral do dente é baixo (Cochrane et al.,2010). Novos estudos sugerem a utilização de dentifrícios remineralizantes, como os dentifrícios de fosfopeptídeo de caseína-fosfato de cálcio amorfo. Embora sejam necessárias mais pesquisas, a literatura já apresenta resultados promissores sobre o uso dessas pastas (Papas et al., 2008; Dashper et al., 2019; Palmier et al., 2020).

Ainda em relação à higiene diária, muitos pacientes oncológicos queixam-se do uso dos cremes dentais. A presença de lauril sulfato de sódio, agente de formação de espuma de dentifrícios, pode remover a camada de mucina e causar úlceras na mucosa oral (Dirix et al., 2006). Outra constante queixa dos pacientes é em relação ao sabor, muitos reclamam que o sabor menta contribui para causar irritação da mucosa (Dirix et al., 2006).

\section{Prevenção por meio de controle profissional}

Por se tratarem de pacientes com alto risco de cárie, recomenda-se que o acompanhamento profissional seja a cada três meses, até que os pacientes apresentem sinais de estabilidade a longo prazo, de forma que visitas semestrais sejam apropriadas. A American Dental Association recomenda a aplicação de flúor tópico em gel ou verniz a cada três meses, em pacientes de alto risco (ADA, 2006). Além disso, as consultas periódicas devem incluir exame clinico preciso, profilaxias e terapia periodontal de suporte. Uma vez ao ano, radiografias periapicais são recomendadas (Noone et al.,2017; Palmier et al.,2020).

\section{Tratamento}

O tratamento restaurador é desafiador. O profissional irá trabalhar em um tecido dentário alterado, no qual a adesão entre a restauração e o substrato irradiado com altas doses de radiação ionizante é complexa (Naves et al., 2012). Do ponto de vista técnico, o atendimento clínico desses pacientes também é dificultado e requer cuidados. Alguns pacientes desenvolvem trismo após a radioterapia, o que limita sua abertura bucal, podendo ser esta a primeira dificuldade que o profissional encontrará para a realização do tratamento (Jawad et al., 2015; Noone et al., 2017; Sroussi et al., 2018; Palmier et al., 2020). Ademais, devido à hipossalivação, a mucosa oral normalmente é sensível, e muitas vezes procedimentos simples como o isolamento relativo se tornam complicados e requerem maior cuidado (Gupta et al., 2015).

A escolha do material restaurador mais adequado para o tratamento das lesões de CRR tem sido outro ponto de discussão. Os materiais restauradores mais empregados são cimentos de ionômero de vidro (CIV) e compósitos resinosos. Os CIV são muito utilizados em pacientes irradiados devido à sua capacidade de liberação de flúor local (McComb et al., 2002; Palmier et al., 2020). Eles também possuem a capacidade de se unir quimicamente à estrutura dentária, devido à conexão entre os grupos carboxil de poliácidos presentes no material e os íons cálcio presentes na estrutura dentária (McComb et al., 2002). 
No entanto, esmalte e dentina irradiados apresentam diminuição da quantidade de cálcio, o que poderia comprometer essa união química (Velo et al., 2018; Miranda et al., 2021). A resistência de união é alterada independente do material restaurador (CIV ou resina), pois a adesão é comprometida se o dente é irradiado previamente à restauração (Yesilyurt et al., 2008; De Moor et al., 2011; Naves et al., 2012; Yadav \& Yadav, 2013; Rodrigues et al., 2018; Palmier et al., 2020).

Quando estes dois materiais são avaliados quanto às suas propriedades mecânicas e químicas, não há alteração química em nenhum deles frente à radiação ionizante (Brandeburski et al., 2018). Porém, os CIV apresentaram maiores alterações nas propriedades mecânicas (Brandeburski et al., 2018) e maiores falhas nas restaurações ao longo dos anos (McComb et al., 2002; De Moor et al., 2011; Galetti et al., 2014). Outro estudo mostrou que restaurações em resina composta apresentaram menores índices de falhas devido à adaptação marginal e forma anatômica quando comparadas ao CIV, em um período de 24 meses (De Moor et al., 2011). Contudo, pacientes que não realizam o uso regular de flúor apresentam melhores resultados nas restaurações em CIV, quando comparada a presença de cáries secundárias, sendo este material indicado para este tipo de pacientes (McComb et al., 2002; De Moor et al., 2011; Moore et al., 2020). Portanto, os ionômeros de vidro (especialmente a formulação convencional) fornecem maior inibição da cárie clínica, mas erodem facilmente, enquanto a resina composta fornece maior integridade estrutural (De Moor et al., 2011). A figura 4 mostra o aspecto imediato após restauração em resina composta em dente irradiado e, em seguida, aplicação de verniz fluoretado. 
Figura 4. Restauração em resina composta nos dentes 41 e 42. Aspecto inicial das CRR. Note lesão cariosa bastante avançada no dente 41 (A). Restauração em resina composta nos dentes 41 e 42 (B). Aplicação de verniz fluoretado (S.S. White, Rio de Janeiro, RJ, Brasil) contendo 22.600 ppm de flúor, 3 aplicações, no intervalo de 7 em 7 dias) (C).


Fonte: Autores.

Em suma, ambos os materiais apresentam vantagens e limitações. Baseado na literatura pertinente, ainda não há um consenso sobre o protocolo restaurador ideal para as lesões de CRR, sendo necessários mais estudos clínicos para a validação desses materiais em pacientes oncológicos de cabeça e pescoço, principalmente a longo prazo. Cabe ao cirurgião-dentista avaliar a situação clínica para determinar a melhor conduta. Em algumas situações, o CIV é utilizado em um primeiro momento como estratégia para adequação do meio bucal e, posteriormente, é feita a substituição por restaurações em compósito resinoso. Entretanto, sabendo das limitações da adesão ao esmalte e dentina irradiados, o acompanhamento e controle frequente desses pacientes no consultório odontológico torna-se fundamental.

\section{Conclusão}

A presente revisão deixa claro que a prevenção e o tratamento das lesões de CRR são desafiadores. Frente às alterações salivares e das propriedades do esmalte e da dentina, o poder de destruição da CRR é evidente. O ambiente oral 
torna-se extremamente cariogênico, com mudanças no $\mathrm{pH}$ e na saliva, muitas vezes acompanhadas de uma higienização deficiente. Assim, vários são os fatores que contribuem para a rápida evolução da cárie e para o insucesso dos tratamentos restauradores. A cooperação do paciente é fator fundamental para o controle dessa doença. Infelizmente, durante e após o tratamento oncológico, muitos pacientes encontram-se desmotivados ou com dor devido à hipossalivação e/ou mucosites, o que gera dificuldades na alimentação e na higienização. Somado a isto, encontra-se o fator psicológico. A aparência dos dentes após o acometimento pela CRR não é agradável esteticamente e isso pode desanimar o paciente, fazendo com que ele não contribua com sua própria saúde bucal. Nesse sentido, é fundamental que o cirurgião-dentista atue como agente motivador do paciente. É importante o conhecimento científico sobre os fatores específicos da CRR, para que o profissional possa explicar as limitações e especificidades de cada caso. O paciente pós-radioterapia não poderá ter alta do consultório odontológico, pelo contrário, deverá ter retornos periódicos para acompanhamento, profilaxia, acabamento/polimento das restaurações e troca de restaurações insatisfatórias. E, mais importante ainda que os retornos, é o cuidado diário que este paciente precisa ter com higienização e uso contínuo do fluoreto em casa, para manutenção da sua saúde bucal. É imprescindível que novas pesquisas sejam realizadas, a fim de investigar métodos de diagnóstico e prevenção cada vez melhores, bem como protocolos de tratamento específicos, buscando acima de tudo restabelecer a saúde e melhorar a qualidade de vida desses pacientes oncológicos.

\section{Referências}

Alves, Y. B., Silva, P. C. H. Da, Carvalho, G. C., Caldas, J. P. M. \& Veloso, H. H. P. (2020). Impact of radiotherapy to the head and neck region on the oral condition. Research, Society and Development, 9(10).

American Dental Association Council on Scientific Affairs. (2006) Professionally applied topical fluoride: evidence-based clinical recommendations. $J$ Am Dent Assoc, 137(8):1151-9.

Bhandari, S., Soni, B. W., Bahl, A. \& Ghoshal, S. (2020). Radiotherapy-induced oral morbidities in head and neck cancer patients. Special Care in Dentistry, 40(3), 238-250.

Brandeburski, S. B. N. \& Della Bona, A. (2018). Effect of ionizing radiation on properties of restorative materials. Dental Materials, 34(2), $221-227$.

Buglione, M., Cavagnini, R., Di Rosario, F., Sottocornola, L., Maddalo, M., Vassalli, L., Grisanti, S., Salgarello, S., Orlandi, E., Paganelli, C., Majorana, A., Gastaldi, G., Bossi, P., Berruti, A., Pavanato, G., Nicolai, P., Maroldi, R., Barasch, A., Russi, E. G., Raber-Durlacher, J., Murphy, B. \& Magrini, S. M. (2016) Oral toxicity management in head and neck cancer patients treated with chemotherapy and radiation: Dental pathologies and osteoradionecrosis (Part 1 ) literature review and consensus statement. Critical Reviews in Oncology/Hematology, 97, 131-142.

Cartaxo, A.C., Marinho, L. C. N., Silva, D. N. D. A., Silva, N. T. Da., Morais, M. De. L. S. De. A., Dorini, A. L. \& Martins, A. R. L. De. A. (2021) Impact of radiotherapy in head and neck regions on periodontal and oral hygiene conditions. Research, Society and Development, 10 (2), e44410212745.

Cochrane, N. J., Cai, F., Huq, N. L., Burrow, M. F., Reynolds, E. C. (2010) New approaches to enhanced remineralization of tooth enamel. J Dent Res, 89:1187-1197.

Comodo, G. V., Palma, L. F., Dos Santos, M. S., Seoantes, G. A., Gonnelli, F. A. S., Segreto, R. A., Segreto, H. R. C. \& Reimão, J. Q. (2020) Opportunistic oral infections in patients submitted to radiotherapy for head and neck cancer: a retrospective study. Research, Society and Development, 9 (3), e164932685.

Dashper, S. G., Shen, P., Sim, C., Liu, S. W., Butler, C. A., Mitchell, H. L., D'Cruze, T., Yuan, Y., Hoffmann, B., Walker, G. D., Catmull, D. V., Reynolds, C., \& Reynolds, E. C. (2019). CPP-ACP Promotes SnF 2 Efficacy in a Polymicrobial Caries Model. Journal of dental research, 98(2), $218-224$.

De Felice, F., Polimeni, A., Valentini, V., Brugnoletti, O., Cassoni, A., Greco, A., de Vincentiis, M. \& Tombolini, V. (2018). Radiotherapy Controversies and Prospective in Head and Neck Cancer: A Literature-Based Critical Review. Neoplasia, 20(3), 227-232.

De Moor, R. J., Stassen, I. G., van 't Veldt, Y., Torbeyns, D. \& Hommez, G. M. (2011). Two-year clinical performance of glass ionomer and resin composite restorations in xerostomic head- and neck-irradiated cancer patients. Clinical Oral Investigations, 15(1), 31-8.

Dirix, P., Nuyts, S. \& Bogaert, W. V. D. (2006). Radiation-Induced Xerostomia in Patients with Head and Neck Cancer: A Literature Review. Cancer, 107(11), 2525-34.

Epstein, J. B., van der Meij, E. H., Lunn, R., Stevenson-Moore, P. (1996) Effects of compliance with fluoride gel application on caries and caries risk in patients after radiation therapy for head and neck cancer. Oral Surg Oral Med Oral Pathol Oral Radiol Endod, 82:268-275.

Fonseca, J. M., Troconis, C. C., Palmier, N. R., Gomes-Silva, W., Paglioni, M. D., Araújo, A. L., Arboleda, L. P., Filho, A. J., González-Arriagada, W. A., Goes, M. F., Lopes, M. A., Brandão, T. B., Vargas, P. A., Ribeiro, A. C. \& Santos-Silva, A. R. (2020). The impact of head and neck radiotherapy on the dentine-enamel junction: a systematic review. Medicina Oral Patologia Oral y Cirurgia Bucal, 25 (1) 96-105. 
Galetti, R., Santos-Silva, A. R., Antunes, A. N., Alves, F. A., Lopes, A. M. \& de Goes, M. F. (2014) Radiotherapy does not impair dentin adhesive properties in head and neck cancer patients. Clinical Oral Investigations, 18(7), 1171-8.

Gonçalves, L. M., Palma-Dibb, R. G., Paula-Silva, F. W., Oliveira, H. F., Nelson-Filho, P. Silva, L. A. \& Queiroz, A. M. (2014). Radiation therapy alters microhardness and microstructure of enamel and dentin of permanent human teeth. Journal of Dentistry, 42(8), 986-92.

Gouvêa Vasconcellos, A. F., Palmier, N. R., Ribeiro, A., Normando, A., Morais-Faria, K., Gomes-Silva, W., Vechiato Filho, A. J., de Goes, M. F., Paes Leme, A. F., Brandão, T. B., Lopes, M. A., Marsh, P. D., \& Santos-Silva, A. R. (2020). Impact of Clustering Oral Symptoms in the Pathogenesis of Radiation Caries: A Systematic Review. Caries research, 54(2), 113-126.

Gupta, N., Pal, M., Rawat, S., Grewal, M. S., Garg, H., Chauhan, D., Ahlawat, P., Tandon, S., Khurana, R., Pahuja, A. K., Mayank, M. \& Devnani, B. (2015). Radiation-induced dental caries, prevention and treatment - A systematic review. National Journal of Maxillofacial Surgery, 6(2), 160-6.

INCA, Instituto Nacional de Câncer José Alencar Gomes da Silva. (2019). Estimativa 2020: incidência de câncer no Brasil. https://www.inca.gov.br/estimativa/estado-capital/brasil

Jansma, J., Vissink, A., Jongebloed, W. L., Retief, D. H. \& 's-Gravenmade, E. J. (1993). Natural and induced radiation caries: A SEM study. American Journal of Dentistry, 6,130-136.

Jawad, H., Hodson, N. A. \& Nixon, P. J. (2015). A review of dental treatment of head and neck cancer patients, before, during and after radiotherapy: part 1. Brazilian Dental Journal, 218(2), 65-8.

Kawashita, Y., Soutome, S., Umeda, M. \& Saito, T. (2020) Oral management strategies for radiotherapy of head and neck cancer. Japanese Dental Science Review, 56 (1), 62-67.

Kırca, K. \& Kutlutürkan, S. (2017) Symptoms of patients with head and neck cancers undergoing radiotherapy. European Journal of Cancer Care, 26 (6).

Kielbassa, A. M., Schaller, H. G., Hellwig, E. (1998) Qualitative Befunde bei in situ erzeugter Initialkaries in tumortherapeutisch bestrahltem Dentin: eine kombiniert rasterelektronenmikroskopische und mikroradiographische Studie. Acta Med Dent Helv, 3:161-168.

Kudkuli, J., Agrawal, A., Gurjar, O. P., Sharma, S. D., Rekha, P. D., Manzoor, M. A. P., Singh, B., Rao, B. S. \& Abdulla, R. (2020) Demineralization of tooth enamel following radiation therapy; An in vitro microstructure and microhardness analysis. Journal of Cancer Research and Therapeutics, 16 (3), 612-618.

Lan, X., Chan, J. Y. K., Pu, J. J., Qiao, W., Pang, S., Yang, W. F., Wong, K. C. W., Kwong, D. L. W. \& Su, Y. X. (2020) Saliva electrolyte analysis and xerostomia-related quality of life in nasopharyngeal carcinoma patients following intensity-modulated radiation therapy. Radiotherapy and Oncology, 150, 97103.

Lopes, C. C. A., Rodrigues, R. B., Cenci, M. S., Uehara, J. L. S., Maske T. T., Limirio P. H. J. O., Soares, P. B. F., Novais, V. R. (2021) Effect of ionizing radiation and cariogenic biofilm challenge on root-dentin caries. Clin Oral Investig.

Lopes, C. C. A., Soares, C. J., Lara, V. C., Arana-Chavez, V. E., Soares, P. B. \& Novais, V. R. (2018). Effect of fluoride application during radiotherapy on enamel demineralization. Journal of Applied Oral Science, 27.

Lou, J., Huang, P., Ma, C., Zheng, Y., Chen, J., Liang, Y., Li, H., Yin, Y., Liu, D., Yu, G. \& Li, D. (2018). Parotid gland radiation dose-xerostomia relationships based on actual delivered dose for nasopharyngeal carcinoma. Journal of Applied Clinical Medical Physics, 19, 251-60.

Lu, H., Zhao, Q., Guo, J., Zeng, B., Yu, X., Yu, D. \& Zhao, W. (2019) Direct radiation-induced effects on dental hard tissue. Radiation Oncology, 14 (1), 5.

Madrid, C. C., de Pauli Paglioni, M., Line, S. R., Vasconcelos, K. G., Brandão, T. B., Lopes, M. A., Santos-Silva, A. R., De Goes, M. F. (2017) Structural Analysis of Enamel in Teeth from Head-and-Neck Cancer Patients Who Underwent Radiotherapy. Caries Res, 51(2):119-128.

McComb, D., Erickson, R. L., Maxymiw, W. G. \& Wood, R. E. (2002). A clinical comparison of glass ionomer, resin-modified glass ionomer and resin composite restorations in the treatment of cervical caries in xerostomic head and neck radiation patients. Operative Dentistry, $27,430-7$.

McGuire, J. D., Gorski, J. P., Dusevich, V., Wang, Y. \& Walker, M. P. (2014). Type IV collagen is a novel DEJ biomarker that is reduced by radiotherapy. Journal of Dental Research, 93(10), 1028-34.

Miranda, R. R., Ribeiro, T. E., Silva, E. L. C. D., Simamoto Júnior, P. C., Soares, C. J., Novais, V. R. (2021) Effects of fractionation and ionizing radiation dose on the chemical composition and microhardness of enamel. Arch Oral Biol.

Miranda, R. R., Silva, A. C. A., Dantas, N. O., Soares, C. J. \& Novais, V. R. (2019) Chemical analysis of in vivo-irradiated dentine of head and neck cancer patients by ATR-FTIR and Raman spectroscopy. Clinical Oral Investigations, 23(8), 3351-3358.

Moore, C., McLister, C., Cardwell, C., O'Neill, C., Donnelly, M. \& McKenna, G. (2020) Dental caries following radiotherapy for head and neck cancer: A systematic review. Oral Oncology, 100, 104484

Müller, V. J., Belibasakis, G. N., Bosshard, P. P., Wiedemeier, D. B., Bichsel, D., Rücker, M. \& Stadlinger, B. (2019) Change of saliva composition with radiotherapy. Archives of Oral Biology, 106:104480.

Muñoz, M. A., Garín - Correa, C., González - Arriagada, W., Davila, X. Q., Häberle, P., Bedran - Russo, A., \& Luque - Martínez, I. (2020) The Adverse Effects of Radiotherapy on the Structure of Dental Hard Tissues and Longevity of Dental Restoration. International Journal of Radiation Biology, 96 (7), 910 -918 .

Naves, L. Z., Novais, V. R., Armstrong, S. R., Correr-Sobrinho, L. \& Soares, C. J. (2012) Effect of gamma radiation on bonding to human enamel and dentin. Supportive care in cancer: official journal of the Multinational Association of Supportive Care in Cancer, 20, 2873-8.

Noone, J. \& Barclay, C. (2017) Head and Neck Cancer Patients - Information for the General Dental Practitioner. Dental Update, 44 (3), $209-215$. 
Novais, V. R., Soares, P. B., Guimarães, C. M., Schliebe, L. R., Braga, S. S. L. \& Soares, C. J. (2016). Effect of gamma radiation and endodontic treatment on mechanical properties of human and bovine root dentin. Brazilian Oral Research, 27(6), 670-4.

Palmier, N. R., Migliorati, C. A., Prado-Ribeiro, A. C., de Oliveira, M. C. Q., Vechiato Filho, A. J., de Goes, M. F., Brandão, T. B., Lopes, M. A. \& SantosSilva, A. R. (2020) Radiation-related caries: current diagnostic, prognostic, and management paradigms. Oral Surgery, Oral Medicine, Oral Pathology and Oral Radiology, 130 (1), 52-62.

Papas, A., Russell, D., Singh, M., Kent, R., Triol, C. \& Winston, A. (2008). Caries clinical trial of a remineralising toothpaste in radiation patients. Gerodontology, 25, 76-88.

Pioch, T., Golfels, D. \& Staehle, H. J. (1992) An experimental study of the stability of irradiated teeth in the region of the dentinoenamel junction. Endodontics and Dental Traumatology, 8 (6), 241-4.

Powell, L. V., Persson, R. E., Kiyak, H. A. (2001) Effect of a 0.12\% chlorhexidine rinse on salivary lactobacilli and mutans streptococci. J Dent Res, 80:159.

Qing, P., Huang, S., Gao, S., Qian, L. \& Yu, H. (2016). Effect of gamma irradiation on the wear behavior of human tooth dentin. Clinical Oral Investigation, 20(9), 2379-86.

Reed, R., Xu, C., Liu, Y., Gorski, J. P., Wang, Y. \& Walker, M. P. (2015). Radiotherapy effect on nano-mechanical properties and chemical composition of enamel and dentine. Archives of Oral Biology, 60(5), 690-7.

Rodrigues, R. B., Soares, C. J., Junior, P. C. S., Lara, V. C., Arana-Chavez, V. E. \& Novais, V. R. (2018). Influence of radiotherapy on the dentin properties and bond strength. Clinical Oral Investigations, 22(2), 875-883.

Roblegg, E., Coughran, A., \& Sirjani, D. (2019). Saliva: An all-rounder of our body. European journal of pharmaceutics and biopharmaceutics: official journal of Arbeitsgemeinschaft fur Pharmazeutische Verfahrenstechnik e.V, 142, 133-141.

Santin, G. C., Palma-Dibb, R. G., Romano, F. L., de Oliveira, H. F., Nelson Filho, P. \& de Queiroz, A. M. (2015). Physical and adhesive properties of dental enamel after radiotherapy and bonding of metal and ceramic brackets. American Journal of Orthodontics and Dentofacial Orthopedics, 148(2), $283-92$.

Silva, A. R., Alves, F. A., Antunes, A., Goes, M. F., \& Lopes, M. A. (2009). Patterns of demineralization and dentin reactions in radiation-related caries. Caries research, 43(1),43-49.

Soares, C. J., Castro, C. G., Neiva, N. A., Soares, P. V., Santos-Filho, P. C. F., Naves, L. Z. \& Pereira, P. N. (2010). Effect of gamma irradiation on ultimate tensile strength of enamel and dentin. Journal of Dental Research, 89(2), 159-64.

Soares, C. J., Neiva, N. A., Soares, P. B., Dechichi, P., Novais, V. R., Naves, L. Z. \& Marques, M. R. (2011). Effects of chlorhexidine and fluoride on irradiated enamel and dentin. Journal of Dental Research, 90,659-664.

Sroussi, H. Y., Jessri, M. \& Epstein, J. (2018) Oral assessment and management of the patient with head and neck cancer. Oral and Maxillofacial Surgery Clinics of North America, 30, 445-458.

Teruel, J., Alcolea, A., Hernández, A., \& Ruiz, A. J. (2015). Comparison of chemical composition of enamel and dentine in human, bovine, porcine and ovine teeth. Archives of oral biology, 60(5), 768-775.

Velo, M. M. A. C., Farha, A. L. H., da Silva Santos, P. S., Shiota, A., Sansavino, S.Z., Souza, A.T., Honório, H. M. \& Wang, L. (2018). Radiotherapy alters the composition, structural and mechanical properties of root dentin in vitro. Clinical Oral Investigation, 22(8), 2871-2878.

Vissink, A., Jansma, J., Spijkervet, F. K., Burlage, F. R. \& Coppes, R. P. (2003). Oral sequelae of head and neck radiotherapy. Critical Reviews in Oral Biology \& Medicine, 14(3), 199-212.

Wu, V. W. C. \& Leung, K. Y. (2019) A Review on the Assessment of Radiation Induced Salivary Gland Damage After Radiotherapy. Frontiers on Oncology, 9,1090 .

Walker, M. P., Wichman, B., Cheng, A. L., Coster, J. \& Williams, K. B. (2011). Impact of Radiotherapy Dose on Dentition Breakdown in Head and Neck Cancer Patients. Practical Radiation Oncology, 1(3), 142-148.

Yadav, S. \& Yadav, H. (2013). Ionizing irradiation affects the microtensile resin dentin bond strength under simulated clinical conditions. Journal of conservative dentistry, 16, 148-151.

Yesilyurt, C., Bulucu, B., Sezen, O., Bulut, G. \& Celik, D. (2008) Bond strengths of two conventional glass-ionomer cements to irradiated and non-irradiated dentin. Dental Materials Journal, 27 (5), 695-701. 